\title{
Creación y operaciones de transformación: aportes para una retórica del diseño
}

\section{Eiriz, Claudio [ver currículum del autor, docente de la Facultad de Diseño y Comunicación]}

\section{Resumen:}

¿Qué es lo que hay en común ente cosas tan disimiles como las mutaciones cromosómicas, las operaciones de superposición de la simetría o la forma musical? La pregunta no es caprichosa. Hay un "aire de familia" entre los mecanismos a partir de los cuales conocemos el mundo y al mismo tiempo nos reconocemos en él. El presente trabajo parte de suponer que el diseño -como todo acto de conocimiento- no es un campo meramente "inspirado". Toda creación implica, entre otras cosas, abrevar en un repertorio de operaciones de transformación que se expresan -como lo supo ver Jaques Durand-en las antiguas figuras de la retórica. En primer término se intentará articular este repertorio de operaciones con conceptos conexos tales como: envolvente proto-narrativa y grado 0 . En segundo término, se esbozará una "tabla de elementos" que dé cuenta de la totalidad de las operaciones posibles. Asimismo, se hará especial énfasis en la retórica del audiovisual y los recursos generadores de la forma musical. Este estudio se inscribe en una tradición que tiene su origen en los trabajos de Roland Barthes acerca de la retórica de la imagen y aspira a contribuir a la construcción de una meta-teoría del diseño y los procesos de creación.

Palabras clave:

Retórica audiovisual - retórica musical - grado cero - Roland Barthes - Jaques Durand - imagen - sonido creatividad - diseño.

El tiempo se hace tiempo humano en cuanto se atiene al modo narrativo; a su vez la narración es significativa en la medida en que descubre los rasgos de la experiencia temporal. (Paul Ricouer) 
¿Qué es lo que hay en común entre cosas tan disímiles como las mutaciones cromosómicas, las operaciones de superposición de la simetría, la forma musical, el diseño audiovisual o la transmisión de conocimientos? La pregunta no es caprichosa. Hace ya muchos años alguien me hizo notar el "aire de familia" que existe entre las operaciones de superposición de la simetría y los recursos generadores de la forma musical. Por ejemplo, una traslación regular es semejante a una repetición en música; tanto en simetría como en música existen las reflexiones especulares; la extensión en simetría es muy similar a la aumentación en música1. Tiempo después me encontré con un histórico artículo de Jaques Durand (1970) acerca de la retórica de la imagen publicitaria. Este autor siguiendo el camino que había trazado Roland Barthes (1964), diseñó un cuadro que ordena las múltiples figuras retóricas con el fin de aplicarlas a la imagen publicitaria. Durand planteó la necesidad de construir una retórica formal que supere los límites de la palabra -algo que parecería viable como analogía para nuestro propósito de explorar las operaciones retóricas en distintos campos del diseño2 . La figura de la retórica -para Durand- puede definirse, como un mecanismo que modifica algunos elementos de la proposición original. De acuerdo al componente que se altere, son posibles dos dimensiones: una de ellas refiere a la naturaleza de la operación que se realiza en tanto la otra, a la naturaleza de la relación que vincula los elementos variables (la proposición de base y la modificada). Se reconocen dos operaciones fundamentales y dos derivadas.

Obtenemos así: la Adjunción, la Supresión, la Sustitución (supresión seguida de adjunción), el Intercambio (dos sustituciones recíprocas)3 . Las relaciones que vinculan los elementos variables son: identidad, similitud, diferencia y oposición4. Luego, y sobre la base de operaciones y relaciones, Durand construye un cuadro de doble entrada. En él ubica las figuras de la retórica y proporciona ejemplos de la imagen publicitaria. Nosotros haremos un cuadro adaptado, tomando como referencia el cuadro de Durand y con el objetivo de hacerlo útil a nuestros propósitos. Ahora bien, antes de tomar como recurso el cuadro que diseñó Durand, considero pertinente hacer una "enmienda" al esquema que propuso. En este sentido, recordemos que este autor entiende que la figura retórica puede definirse como un mecanismo que modifica algunos elementos de la proposición original o de base. Estamos de acuerdo con esta definición pero debo aclarar que sustituiré la idea de "proposición de base" por la de "grado cero". Más allá de las discusiones acerca de la definición de grado cero, entendemos este concepto como el elemento o situación esperados (es decir anticipado, concebido) en determinado sitio de un enunciado, en virtud de una estructura particular de ese enunciado cualquiera sea el nivel de integración del mismo. Hay que hacer notar que tal como lo ha teorizado el Grupo $\mu$ (p. 238), existe el grado concebido (grado 0) y el grado percibido. Se espera ese elemento (grado concebido) y esto que se espera puede confirmarse o quedar frustrado. En su lugar se presentará otra cosa diferente (grado percibido). Ese otro elemento tendrá una relación de identidad, similitud, diferencia u oposición con el grado concebido.

Consideramos que la inclusión del concepto de grado cero y sus categorías conexas son de gran ayuda para entender la cuestión de las operaciones de transformación que constituyen las figuras retóricas. En especial en las artes temporales y en lo que concierne a la narratividad, se hace insoslayable incorporar el tema de la generación de expectativas, su confirmación o frustración. Este esquema teórico es importante articularlo con la noción de "envolvente" proto-narrativa (EPN). Así, todo evento musical o audiovisual y toda forma temporal despierta expectativas acerca de cómo podría continuar, es decir, cómo "gatilla" en el sujeto, la competencia que le permite anticipar y en consecuencia, generar una intriga. Este contorno dinámico, esta EPN en su devenir -al estar orientada hacia un objetivo- nos conduce a un punto fijo, un punto de máxima tensión, un punto de explosión, de ruptura temporal. No hay duda de que la EPN lleva dentro de sí el presentimiento de este clímax. 
Ese encadenamiento de acontecimientos por el que nos dejamos llevar pareciera estar organizado conforme a un fin que tiende a realizar. Estas expectativas pueden cumplirse o frustrarse. A este contorno temporal, Imberty (2012) lo denomina "envolvente proto-narrativa" (EPN)5 . La EPN es un contorno de afectividad repartido en el tiempo y orientado hacia un objetivo.

Es, por lo tanto, una forma proto-semiótica de la experiencia interior del tiempo, una matriz del "relato" de las tensiones y de las distensiones ligadas a la "intriga" (...) de la búsqueda de una satisfacción, que da a la experiencia su unidad global, sea cual fuere el grado de complejidad. (Imbrety, 2012)

El citado autor plantea también que la música y cualquier forma narrativa está "calcada" sobre esta dinámica que, en última instancia, tiene que ver con la dinámica del deseo en tanto compromete a la vivencia del tiempo. "Y otra cosa que debemos decir de la EPN, es que es básicamente de naturaleza temporal, es una forma únicamente temporal del relato, anterior al cual se van a insertar contenidos diversos" 7 . (Imberty, 1998)

\section{II}

¿Puede el cuadro de Durand ir en auxilio de otros campos de conocimiento tales como las mutaciones genéticas, las diferencias entre los contenidos a enseñar y los efectivamente enseñados o los lapsus calami? Yo no tengo la respuesta. Sin embargo, sólo para demostrar que la idea no es descabellada -y antes de explorar las operaciones en el audiovisual y la música- daré algunos ejemplos. Las mutaciones cromosómicas, por ejemplo, constituyen modificaciones de grandes segmentos de ADN. Existen distintos tipos de mutaciones. Cuando se pierde un cromosoma se denomina "Delección" (elipsis). Si un segmento de cromosoma se halla "repetido" se lo denomina "Duplicación". Se denomina "Inversión" cuando un segmento de cromosoma gira sobre sí mismo. Hay "Translocación" cuando un segmento de ADN pasa de un cromosoma a otro. Las mutaciones genéticas siempre implican la "Sustitución" de un nucleótido por otro. (Cardona, 2002) Creo que estos ejemplos bastan para hacernos una idea de que las operaciones de adjunción, supresión, sustitución y permutación son las que están en juego. En la transmisión de contenidos de enseñanza ocurre algo parecido a la mutación genética -pero esta vez no es el genoma humano el que se ve afectado en la replicación sino el genoma cultural-: el contenido sufre una serie de transformaciones que lo hacen apto para coexistir en el sistema didáctico. Ives Chevallard (1991), quien desarrolla la teoría de la transposición didáctica plantea que los contenidos sufren creaciones de objeto y sustituciones patológicas. Se podría decir que se crean tanto metáforas como metonimias. Sin embargo, si nos detenemos en los detalles de las diferencias entre el contenido designado a enseñar y el efectivamente enseñado, es posible observar una cantidad de operaciones compatibles con las propuestas por el cuadro al que estamos haciendo referencia. Lo mismo ocurre con el lapsus calami. Cualquiera comete errores de escritura. En algunos casos estos errores no son causados por una mera distracción, son recurrentes y comúnmente se los llama dislexia. Se hace posible ordenar los "errores" de la escritura en este cuadro de operaciones y relaciones. Por sólo dar algunos ejemplos observemos las inversiones verticales, inversiones horizontales, repeticiones de letras o sílabas, sustitución de fonemas sordos y sonoros (p-b; t-d; kq/g), etc. (Gonzales, 1999; Pomier, 1993) La hipótesis de este trabajo es que cualquier parcela de la realidad que tenga como objeto de estudio a las transformaciones puede hacerse inteligible a partir de una "tabla de elementos" que distribuye los "mundos posibles" de esas transformaciones. Creemos que las figuras de la retórica son la sedimentación de la historia de los intercambios de los hombres entre sí y con las cosas. En virtud de ello, tenemos la esperanza de que el diseño de un cuadro que formalice las distintas operaciones y relaciones de transformación sea útil tanto como "a priori de inteligibilidad" de las transformaciones, cuanto una 
"caja de herramientas" para la creación. Sin embrago, en este artículo me centraré en dos casos específicos que pertenecen al campo del diseño: el audiovisual y la música.

\section{La retórica del audiovisual}

El estudio de la retórica en el audiovisual no es nuevo. Sólo por dar un ejemplo podemos remitirnos a los trabajos de Joost, G.; Scheuermann, A. (2006) y Joost, G.; Buchmüller, S.; Englert, R. (2008). En estos trabajos se hace una articulación entre secuencias de audiovisual y figuras retó- ricas, se demuestra la pertinencia de esta relación y se dan ejemplos de ello. De todos modos, no basta con hacer una colección de figuras retóricas y demostrar su relación con el audiovisual. Para avanzar se hace necesario integrar estos mecanismos de transformación en un sistema operatorio de estructuras de conjunto. Ese fue mi intento en el proyecto Retórica de las relaciones entre imagen y sonido (Eiriz, 2011). Lo que sigue remite a algunos aspectos de ese trabajo. Tomemos solo tres ejemplos con el objeto identificar algunas figuras retóricas en el seno del cuadro de operaciones y relaciones propuesto.

Casi al final del Film American beauty, vemos al protagonista mirando una fotos, sentado a la mesa de la cocina. En un primer plano de su cabeza, de perfil, vemos una pistola que apunta a su nuca. Un paneo hacia la izquierda nos hace ver un florero con unas rosas rojas y luego una pared blanca. Escuchamos la detonación al tiempo que vemos una mancha de sangre en la pared. La secuencia podría haberse resuelto de otros modos:
a) El sonido del disparo coincidiendo con un fundido a negro (sustitución). Esto constituiría una "reticencia" (casilla 12). Lo que deberíamos haber visto queda oculto por el fundido a negro que, en este caso, no es un mero "signo de puntuación" (punto y aparte), sino la decisión del realizador de ocultar la imagen.

b) Primer plano de la cabeza de perfil y la pistola, luego un corte neto y el sonido del disparo en sincronía con la mancha de sangre en la pared: Nuevamente aquello que esperamos ver es sustituido. Lo que se sustituye en este caso es la causa por el efecto. Deberíamos haber visto el disparo en la cabeza, sin embargo vemos el efecto: la sangre en la pared. Esto constituiría una metonimia (casilla 11).

Sin embargo en el original, la secuencia se estiliza. Digamos que se produce una figura: suspensión (casilla 7). El paneo opera como recurso para suspender la resolución de la escena. Pero además, hay allí figuras de comparación (casilla 10). La cabeza que desde el punto de vista de la forma es un círculo, se asocia a las flores rojas que también son un círculo. Además, este círculo rojo que constituyen las flores, se asocia a la forma y el color de la mancha de sangre que es roja y circular. En una secuencia del cortometraje Hostage, de John Woo, un hombre tiene secuestrada a su amante y pide rescate por ella. Al ser sorprendido por un grupo de policías, toma su revólver (al que le había puesto una sola bala) y se dispara. El tiro no sale. Prueba una segunda vez en este juego de ruleta rusa- y por fin oímos la detonación. En el momento en que la oímos, el "découpage" muestra la impresión que esto le causa a Clive Owen, quien intentaba convencer al raptor de que no se disparara. Nuevamente la escena se podría haber resuelto de modo más breve. Sin embargo, aquí también con el objeto de aumentar la tensión- se produce una figura de repetición (casilla 1): el raptor se dispara dos veces. En la primera, lo que anticipábamos ver queda frustrado. En la segunda oportunidad, el disparo se realiza aunque no vemos las consecuencias del disparo en el suicida, sólo escuchamos el sonido de la detonación y la consecuencia que produce en quien observa la escena. El gesto de Clive Owen espeja la reacción que habríamos tenido si hubiéramos visto la escena sin intermediarios. Nuevamente hay aquí suspensión (casilla 8), 
una sustitución de la causa por el efecto (casilla 11) y probablemente una intercambio a modo de reflexión especular (casilla 13), además de la repetición ya citada. En una secuencia del film The River Wild, a orillas de un río se encuentra una familia: el padre (David Strathairk), la madre (Meryl Streep) el hijo y su perra Magie y dos asesinos (Kevin Bacon y su compañero) que habían matado y enterrado a una víctima. La escena comienza cuando la perra Magie escapa al bosque y corre hacia donde, presuponemos que los asesinos habrían enterrado a la víctima. De inmediato, el Padre (David Strathairk) va detrás de ella. En ese momento, Kevin Bacon hace una seña con la cabeza a su compañero y en ese punto la música de foso se inicia con un sonido sostenido y grave de cuerdas. En cada cambio de plano y a medida que el padre es seguido por el delincuente, se va agregando información sonora que connota suspenso. Primero se agregan algunas notas agudas y luego acordes estridentes. Los mismos, que no siempre coinciden con los cortes en la imagen, realizan una suerte de desplazamiento rítmico (casilla 11). La envolvente general de la banda sonora va aumentando en intensidad. La escena se tensa. La perra parece haber encontrado algo y escarba la tierra. Los gritos del niño llamando a su perra se escuchan en primer plano, fuera de cuadro. La música realiza un crescendo, pero antes del tiempo en que estimábamos que iba a coincidir el clímax de tensión de la banda sonora con la imagen de la perra encontrando el cadáver, un acorde Fortísimo se sincroniza con un plano corto del padre asustado y sorprendido -y por corte- con un plano del asesino que lo perseguía. Luego, un silencio profundo. El asesino pronuncia la palabra "dogs". Los dos personajes se alejan en contraplano. Se escucha el sonido de un insecto. Aquí tenemos también una envolvente: todo ese gran arco de tensión y su Climax evitado, el momento que estimábamos que iba a llegar el climax queda elipsado (casilla 5), el punto fuerte llega antes. Hay una figura de anticipación (casilla 5). Lo que esperábamos ver, se frustra. Luego, con el silencio, se produce una descarga. Aunque el sonido inquietante del insecto nos advierte que la cosa allí no ha terminado. Con estos ejemplos hemos inventariado algunas figuras retóricas alrededor de los puntos de sincronización (PDS)8 en el marco de la envolvente protonarrativa, tal como se ha conceptualizado. Sin embargo, una mera colección de ejemplos parecería no ser suficiente. Es necesario -como hemos dicho- diseñar una tabla de las figuras de la retórica, de modo tal, que nos sirva como herramienta de observación.

Todas las figuras incluidas en el cuadro implican operaciones que se aplican a los diferentes niveles de integración. Es decir que se pueden aplicar tanto a las grandes formas, como a las secuencias, sub-secuencias, etc. En el cuadro desarrollado por Durand, que hemos tomado como analogía, los distintos niveles de integración jerárquica están descriptos en un mismo plano. Nuestro cuadro adaptado a la retórica audiovisual tendrá el mismo espíritu que el de Durand. Para nombrar las transformaciones hemos alternado términos retóricos con términos propios del lenguaje audiovisual. Para esto último hemos tomado como referencia el libro Cómo analizar un film, de Cacetti, Di chio. (1991).

IV. Las figuras de la retórica de la música y los recursos generadores de forma

Clemens Kühn y los recursos generadores de forma musical

En el libro de Clemens Kühn, Tratado de la forma musical (1992) encontramos un primer capítulo titulado "Conformación y coherencia", donde el autor plantea la noción de "recurso generador de forma".

La forma musical -el diseño acabado de una idea, de una parte de una pieza, de toda una composición o de una serie de composiciones- presupone el acto generador de dar forma. Sólo el modelado consciente transforma una serie de notas en los más diversos tipos de manifestaciones inteligibles, crea relaciones entre las partes o 
hace que se enfrenten ásperamente; en distintos momentos y de diferentes maneras, ese modelado apunta a una relación y a una coherencia tanto de los detalles musicales como del conjunto (Kühn, 1992, p. 17)9

Kühn en la música, al igual que Durand en la imagen publicitaria, entienden que la creación no es un campo puramente inspirado e intenta formalizar el conjunto de los recursos creativos.

Kühn denominará a esas acciones que el compositor realiza para dar "forma y coherencia" como recursos generadores de forma. Y señalará una nómina de los mismos, aclarando que éstos, aun "no dicen nada sobre su significado, función y efectos concretos". Que es lo mismo que decir que estos recursos son aún, un conjunto de categorías puras débilmente conectadas y aún no dicen nada acerca de la construcción de una operatoria estructurada. Los recursos que señala este autor son: repetición, variante, diversidad y contraste. Agrega un cuarto recurso - "la carencia de relación"- que no describiremos en este trabajo10. Veamos cómo describe Kühn, estos primeros cuatro recursos y acto seguido, cómo creemos que serían nominados por la antigua retórica musical. De este modo, estaremos poniendo en correlación las figuras de la retórica musical con conceptos del análisis musical que no necesariamente han sido pensados desde la retórica.

\section{Repetición}

Se retoman ideas y partes sin modificación. Las ideas musicales (motivos, frases, etc.) son iguales las unas a las otras. En otros términos, se adjuntan cosas idénticas. Sin embrago, Kühn toma la repetición en un sentido muy general y en parte impreciso. En el mismo ejemplo que nos acerca para dar cuenta de la repetición, al describir el fragmento y casi sin advertirlo, el autor nombra otras figuras (o "recursos" si nos ajustamos a su vocabulario). El autor extrae un fragmento de una obra de Schumann para ilustrar la repetición. La obra es el "Impromptu para piano op. 24" (Hojas de álbum). (Figura 1) Y escribe: "los compases primero y segundo son idénticos, las voces graves de las respectivas segundas mitades de compás invierte, con sus (semicorcheas) complementarias, el movimiento melódico"11. (Kühn,1992, p. 18-19) En efecto, los dos primeros compases son idénticos (Casilla 1), pero en cada compás hay una inversión (casilla 16). Es decir, que en el fragmento no sólo hay repetición (adjunción de identidades) sino, también inversión en forma de espejo. Por lo tanto, en este segundo caso, no se realiza una adjunción de algo idéntico, sino que se está sustituyendo un diseño melódico, por su opuesto, en términos de ejes de simetría. Lo cual nos haría pensar además en una figura de contraste (casilla 4). Es decir que ya en este ejemplo que da cuenta de un compás que se repite, en un nivel de integración inferior observamos más operaciones que constituyen otras figuras. La operación de sustitución y las figuras (o recursos) de inversión y contraste. Si esto fuera analizado en términos de la retórica musical, diríamos que el segundo compás es una "Repetitio", es decir, una repetición de la frase o motivo de apertura en un pasaje sucesivo. Pero además, si tomamos el compás mismo como unidad de análisis, nos encontraremos con que la serie de cuatro semicorcheas es un "Groppo", es decir, un motivo de cuatro notas en forma de arco y que tiene como nota en común la primera y tercera. Con respecto a la inversión, ya en la retórica musical del barroco se hablaba de "Hypallage", es decir, la inversión del tema de la fuga. Si bien esto era utilizado para las formas propias del barroco y no del romanticismo, creemos que en términos de operaciones tiene las mismas características y por lo tanto, es posible de ser generalizada12.

\section{Variante}


Se modifican ideas y partes; son similares entre sí. Se adjuntan similitudes. (Figura 2) Aquí kühn (pp. 24) presenta dos fragmentos de "Sonatas con reexposición modificada" de Carl Philipp Emanuel Bach de 1760. Presenta los primeros cuatro compases de modelo y un fragmento de la versión modificada. Esta última mantiene el curso armónico y la animación de notas melódicas nucleares, pero introduce en estas últimas una "sensible animación rítmica". Esto lo logra adjuntando notas, creando una suerte de circunlocución melódica (casilla 10). En términos de retórica musical denominaríamos a esta figura con el nombre de "Subsumptio" o "Quaesitionotae" (Cercar della nota). A este "rodear la nota", hoy por hoy, lo llamamos bordadura. Si bien este concepto está en relación al "adorno" que se le hace a un tema, su sentido es muy distinto según el contexto en que está inserto. En un tema con variaciones, como lo es el ejemplo presentado, este "Cercar della nota", no es un mero adorno, es el procedimiento mismo que engendra una forma. El hecho de circunvalar las notas nucleares produce el efecto de una variación del modelo.

\section{Diversidad}

Las ideas y partes pujan por apartarse unas a otras sin ser idénticas o sin contrastar marcadamente; son diferentes. Se adjuntan diferencias. (Figura 3) Aquí se presentan tres fragmentos melódicos - rítmicos de un "Ave María" de Josquin des Prés.(1440-1521). Se observa en estos fragmentos la diversidad rítmica. En el primero, las figuras disminuyen progresivamente en duración; en el segundo el uso de la síncopa y en el tercero un cambio de métrica (de cuatro tiempos a tres). Lo que intenta demostrar este autor es que en este caso las partes no son ni similares, ni opuestas entre sí; simplemente son diferentes. En términos de retórica musical denominaríamos a los dos primeros casos, con los nombres de: "Diminitio" o "Meiosis" -es decir, la subdivisión de notas largas en notas de menor duración-, y "Syncope", es decir, una suspensión ordinaria, respectivamente. No hemos encontrado hasta el momento, ninguna referencia en la retórica musical al tercer punto.

\section{Contraste}

Las ideas y partes pujan por apartarse unas de otras y se enfrentan entre sí. Son mutuamente opuestas. En términos de operaciones y relaciones, se adjuntan oposiciones. (Kühn, 1992, p. 26) (Figura 4) Kühn, en este caso, nos da como ejemplo un pequeño fragmento del "Cuarteto de cerdas en la m, KV 464, primer movimiento de W. A Mozart. En el ejemplo se observa claramente como se contraponen línea (compases 1-2 y 5 -6) con acorde (compases 3-4 y 7-8), es decir, hay un contraste textural entre monodia y composición acórdica. Además, el contraste se observa en qué el compás 1 desde la tónica (reposo) conduce a la dominante (tensión) y del 5 al 8 conduce a la tónica (reposo). En términos de retórica musical denominaríamos a esta figura con el nombre de "Antithesis" o "Contrapositum", es decir, una expresión musical de oposición ya sea de: carácter, armónica, o de material temático, en otros aspectos posibles. (casilla 4)

¿Por qué motivo las figuras de la retórica pueden constituir un antecedente de las operaciones de transformación en música? Kühn plantea "Recursos" generadores de forma. Pero los recursos en tanto tales son producto de un "curso". En otras palabras, las estructuras que plantea Kühn, son el producto de una génesis. Y esa génesis es tributaria de ciertas operaciones de transformación de un material dado. Las figuras de la retórica pueden ser pensadas como un mecanismo que modifica algunos elementos de una proposición original. Es decir, que pueden ser pensadas entonces, como mecanismos de transformación13.

Figuras de intercambio Webern. Una analogía con los ejes de simetría 
Demos un salto en la historia. Algunas operaciones -como hemos dicho- no tienen un lugar en el seno del cuadro de las figuras de la retórica musical. Si bien hablamos de la inversión, lo hemos hecho ajustándonos al "Hypallage" utilizado en la fuga. Generalicemos entonces este concepto bajo las figuras que resultan de la operación de "intercambio". El intercambio puede definirse como dos sustituciones recíprocas. Comencemos por un caso que no nos presentará demasiadas dificultades. Sólo bastará recordar las figuras de retrogradación, inversión e inversión retrogradada utilizadas antes de Bach, pasando por Webern y hasta nuestros días. Los ejemplos proliferan por todas partes. Bastará con pensar en los ejes de simetría para visualizar qué es lo que se quiere significar con intercambios. Sólo como ejemplo, observemos los primeros compases del OP 27 de A. Webern (1936) (Variaciones opus 27) (Figura 5) Estos primeros siete compases están constituidos por dos planos superpuestos. El plano superior está construido por la serie original; el plano inferior es la retrogradación (casilla 16) de esta serie. Y aquí ya encontramos una operación de intercambio. Un intercambio que superpone las series con una relación de oposición formal. Si observamos el ritmo, también encontramos que se intercambian dos grupos. Un grupo de corchea con puntillo - semicorchea, con otro grupo conformado por dos semicorcheas. En este caso hay un trocado (aquello que estaba en el plano superior pasa al inferior y viceversa). Por otra parte, en el compás cuatro, hay una retrogradación que involucra el total del fragmento. Los compases 1,2,3 y la mitad del 4, se espejan desde la mitad del compás 4 y compases 5,6 y 7. A su vez, en este mismo punto, hay otro trocado: la serie superior pasa a la mano izquierda y la serie inferior pasa a la mano derecha.

La elipsis

Daremos un ejemplo de elipsis (“Ellipsis"). La elipsis es la omisión de algo esperado. No lo confundiremos aquí con la sinécdoque, que es la parte por el todo y pertenece a otro tipo de figura. (Casilla 5) (Figura 6) El ejemplo es de la "Sonata en La m KV 310, presto de W. A Mozart. Observamos que en este fragmento, los primeros 8 compases resultan ser el modelo, y los 7 compases siguientes una repetición a la cual se le ha e suprimido el compás 5. Y aclaramos que sólo se le ha suprimido y no sustituido por ninguna otra cosa. En este caso, la operación que subyace es la supresión y no la sustitución. Esta última implicaría suprimir algo y poner otro algo en su lugar, incluyendo también un silencio14. Como se habrá notado, ya podemos ir ordenando algunas figuras retóricas musicales (o "recursos" según Kühn) en el seno del cuadro trazado por Durand que hemos de tomar como analogía. Si recordamos los cuatro recursos generadores de forma planteados por Kühn y observamos la primera columna del cuadro de Durand, nos sorprende la coincidencia. Cruzando la operación de Adjunción con las relaciones de identidad, similitud, diferencia y oposición, obtendremos por otro camino, el mismo resultado con el que se encontró Kühn. Repetición (adjunción de identidades); Variante (adjunción de similitudes); Diversificación (adjunción de diferencias) y Contraste (adjunción de oposiciones). Notemos también que lo referente a la columna de operaciones de Intercambio, ya hemos dado ejemplos con las figuras de inversión; retrogradación; inversión retrogradada y trocado. También hemos aludido a la columna de la operación Supresión. Hemos dado un ejemplo de Elipsis, que sería el caso general de figuras de supresión. En adelante es necesario el intento de encontrar ejemplos para llenar todas las casillas del cuadro.

\section{Consideraciones finales}

Las operaciones que hemos intentado describir (y que las figuras retóricas expresan) son acciones que hacemos diariamente para realizar cualquier intercambio con las cosas y con los otros aunque, sin embargo, difíciles de atrapar. Es por eso que el análisis musical por ejemplo -a lo mejor por la dificultad que implica nombrar el 
devenir- apenas las haya tenido en cuenta. Al querer atrapar la mecánica de la música, no se ha hecho más que formar agrupamientos y en general se ha dejado afuera su operar mismo. De todos modos, a pesar de lo difícil que es reducir en conceptos ese "operar" (la idea de "ir transformándose"), nos hemos contentado por el momento, y aunque menos sea para poder hablar de eso, con recurrir a las figuras retóricas, esas viejas hermanas en la historia sedimentada del interactuar humano.

No nos interesa tanto el contenido como las formas. En otras palabras, lo que se intenta es describir de qué modo los contenidos pueden adoptar una determinada forma. Más allá de los contenidos particulares nos centramos en la forma que han adoptado las reglas operatorias a partir de las cuales nos apropiamos del mundo al tiempo que nos reconocemos a nosotros mismos. En principio hay que hacer notar que estas acciones (operaciones) no son meramente psicológicas, antes bien, son acciones descentradas. Están situadas, por decirlo así, en eso que Lacan denominó "Gran Otro" historia de la comunidad sedimentada. Es allí, en esa "Otra escena" donde se alojan las reglas operatorias con las que interactuamos con los otros y con las cosas. De algún modo, aunque pensemos que somos nosotros los que operamos conscientemente, esas reglas ya están allí a la espera de ser accionadas. Es por eso que no hemos arriesgado en la introducción de este escrito a vincular esta "tabla de elementos" de operaciones como un "a priori" de campos de estudios diversos. Por último, es necesario hacer notar que esa maquinaria de operaciones posibles descriptas de modo discontinuo, sólo adquieren continuidad en la medida en que se articulan con los rasgos de la experiencia temporal. En este sentido, es que Michel Chion -al referirse al tema de la anticipación y las expectativas que provoca una secuencia audiovisual- escribe que un movimiento de cámara, un ritmo sonoro o con una evolución de uno de los actores, desencadenan en el espectador un movimiento de anticipación, es decir, se va a generar una expectativa que será confirmada o negada por el paso posterior. Continúa: "esta es la dinámica según la cual funciona una secuencia audiovisual" (1993, p. 58). Y más adelante agrega: "pues no se cansa uno de anticipar y sorprender la anticipación: es la dinámica misma del deseo" (1993, p. 59).

Notas

1. Tomo el clásico libro Forma y simetría (Wolf y Khun , 1977).

2. En la segunda mitad del siglo XX los estudios acerca de la retórica son retomados y enriquecidos por la semiología. Desde que Roland Barthes (1964) propuso la posibilidad de aplicar los estudios de la retórica a la imagen, el intento de crear una retórica formal que supere los límites de la palabra no ha cesado. Así lo atestiguan los trabajos emprendidos por Jacques Durand (1970) y el grupo M (1994), entre otros. De allí en más se ha abierto un camino para la aplicación de los estudios de la retórica formal a otros dominios como la retórica de la tipografía Gamonal Arroyo, R (2006); retórica del marco, Echageray, M y Pastor de Samsó, E. (2008), entre otros.

3. Es importante hacer notar que estas operaciones reenvían a acciones muy simples. Pensemos que un niño muy pequeño es capaz, en sus juegos, de poner un bloque encima de otro. Sustraer algo de un conjunto de elementos; sustraer un objeto de un conjunto de elementos y poner otro objeto en su lugar; intercambiar elementos. Si bien estas acciones son simples, son operaciones si y sólo si, son acciones interiorizadas en estructuras de conjunto, tal como lo ha planteado Jean Piaget. Con esto queremos significar que las operaciones, en tanto tal no son meras acciones aisladas, sino que son componentes de estructuras de grupo que el cuadro que este trabajo se propone diseñar intentará figurar. 
4. Si se realiza una acción, la misma siempre se hace sobre un objeto o sobre el estado de alguna cosa. Es decir las operaciones son transformaciones o procesos. Para ejemplificar diremos que un niño puede sustituir una cosa por otra. Como hemos dicho anteriormente saca (suprime) un objeto de un conjunto de objetos de la misma especie y agrega ( adjunta) en su lugar otro. Entre el primer objeto y el segundo pude haber distintos tipos de relaciones: Identidad: Lo que pone es igual al que ha sustraído (saca un bloque rojo y pone otro bloque rojo). Similitud: Lo que pone es similar al que ha sustraído (saca un bloque rojo y pone un bloque verde. Las demás cualidades permanecen constantes). Diferencia: Lo que pone es distinto del que ha sustraído (saca un bloque rojo y pone una pelota). Oposición: Lo que pone es opuesto al que ha sustraído. Es decir es un paradigma de sólo dos términos (saca un bloque blanco y pone un bloque negro. Suponiendo que sólo hay bloques blancos y negros y las demás cualidades permanecen constantes).

5. Término que toma de Stern, D. (1990).

6. Es por lo tanto, una forma de proto-semiótica de la experiencia interior del tiempo, una matriz del "relato" de las tensiones y de las distensiones ligadas a la "intriga" (Imberty, p. 2012).

7. En su libro Entre el diván y el piano (2006), Gilbert T. Rose lo explica de otro modo: "Los patrones motores de la tensión y la descarga reales que acompañan a las emociones son el extremo cinestésico y corporal del puente que conecta más adelante con la tensión y la descarga virtuales incorporadas en la estructura del arte" (2006, p. 199).

8. EI PDS forma parte del fenómeno general de la síncresis, pero constituye un momento muy especial de encuentro entre un instante sonoro y un instante visual. Este momento "relevante", está más "acentuado". Digamos mejor, que ocupa un lugar de jerarquía en el "fraseo" del film. Chion en la audiovisión (1993, p. 61) hace la analogía entre el PDS y el acorde musical, que es lo que "acusaría lo simultáneo en música".

9. El subrayado es nuestro.

10. Consideramos que la "carencia de relación" de la que habla Kün se podrá integrar al cuadro de operaciones que tiene como uno de sus propósitos en presente trabajo.

11. El subrayado es nuestro.

12. Cabe hacer aquí una aclaración. La idea de generalización del concepto de figuras retóricas, es un propósito que perseguiremos en todo este trabajo. Tomamos la idea de "generalización del concepto" tal como lo hace Ausubel, en el sentido de conceptos inclusores, que ofrezcan la posibilidad de ver en estos esquemas de acción $\mathrm{u}$ operaciones y en esas configuraciones sonoras resultantes, o figuras, el origen de los actos que crean una obra musical.

13. Las figuras de la retórica han estado históricamente implicadas, tanto con la composición musical como con el análisis (como herramienta prescriptiva para la composición y herramienta de análisis), es fácil inferir que partir de ellas, estudiar las transformaciones, es un punto de partida genuino. Pero también, tomar los estudios de la retórica musical como antecedente de las trasformaciones en música, no está exenta de inconvenientes. En principio, -como escribe Cartas Martín (2005)- existiría un conflicto terminológico; no hay una doctrina claramente definida para el barroco y toda la música posterior al mismo, a pesar de que con frecuencia se aluda 
a las figuras de la retórica en variados análisis. Además, como hemos notado, no todas las operaciones tienen un correlato en las figuras de retórica musical. De todos modos los estudios históricos acerca de la retórica musical podrán servir como insumo para contrastar con un cuadro formal operatorio de transformaciones en música. Ver también Lopez Cano (2012).

14. La pregunta acerca del límite entre lo que es idéntico o similar estará dado por el contexto. Como se ha dicho, no se pueden tomar las operaciones como acciones aisladas, y agregamos aquí que tampoco las relaciones están dadas a priori. Hemos sugerido que los limites por encima del cual algo es diferente y por debajo del cual algo es semejante está dada por el contexto. En este sentido es que decimos que las operaciones son acciones interiorizadas en estructuras de conjunto.

\section{Bibliografía}

Benveniste, E. (1985). Problemas de lingüística general II (6a. ed.). México: Siglo XXI editores. Cacetti, F. y Di chio, F. (1991). Como analizar un film. Barcelona: Paidos. Cardona, L. (2001). Genética: de Darwin al genoma humano. Barcelona: Océano. Chevallard, I. (1995). La transposición didáctica. Buenos Aires: Aique. Chion, M. (1993). La audiovisión: introducción a un análisis conjunto de la imagen y el sonido. Barcelona: Paidos. Groupe $\mu$. (1993). Tratado del signo visual. Madrid: Cátedra. Imberty, M. (1998). Ponencia realizada por Michel Imberty, en la Licenciatura en Musicoterapia - Universidad Abierta Interamericana. Buenos Aires. Wolf, K. y Kühn, D. (1977). Forma y simetría (4a ed.). Buenos Aires: EUDEBA. Khün, C. (1992). Tratado de la forma musical. Barcelona: Editorial Labor. Pomier, G. (1993). Nacimiento y renacimiento de la escritura. Buenos Aires: Nueva visión. Lopez Cano, R. (2012). Música y Retórica en el Barroco. Ed. Amalgama. Saitta, C. (2002). El ritmo musical. Buenos Aires: Saitta Publicaciones musicales. Stern, D. (1991). El mundo interpersonal del infante. Buenos Aires: Paidós. Torrance, J. (1977). "Cómo evocar la actividad creadora de la mente en los grados primarios" en Educación y capacidad creativa. Madrid: Morava.

\section{Medios electrónicos}

Barthes, R. (1964). Rhétorique de L'image. Communications, 4, 40-50. Recuperado de: http:// www.oasisfle.com/doc_pdf/roland_barthes_rhetorique_image.pdf Cartas Martín, I. (2005). Retórica musical. El madrigal "Lo pur respiro de Carlos Gesualdo. ICONO 14. n 5, Revista de comunicación. Recuperado de: http://www.icono14.net/ revista/num5/articulo8.htm Durand, J. (1970). Rethórique de I'image publicitaire. Communications, 15, 70-95. Recuperado de: http://pagesperso-orange.fr/jacques.durand/Site/Textes/t9.htm

Echageray, M. y Pastor de Samsó, E. (2008). El espacio en el Diseño: Una interpretación de Retórica del Marco del Groupe M desde el diseño gráfico. Revista Huellas, Búsquedas en Artes y Diseño, $n^{\circ} 6$, Mendoza, Argentina, ISSN 1666-8197. Recuperado de: http://bdigital. uncu.edu.ar/objetos_digitales/2524/echegarayhuellas6-08.pdf Eiriz, C. (2011). Retórica de las relaciones entre imagen y sonido: Un estudio acerca de las operaciones y relaciones en los puntos de sincronización. Universidad de Palermo. Recuperado de: http://fido.palermo.edu/servicios_dyc/proyectograduacion/archivos/994.pdf Gamonal Arroyo, R. (2006). TIPO/RETÓRICA. Una aproximación a la Retórica Tipográfica. Icono 14 / revista de comunicación y nuevas tecnologías. ISSN: 1697-8293. Recuperad de: http://www.icono14.net/revista/num5/articulo6.htm Guerri, C. (2007). Simetría como retórica de la forma. Trabajo presentado en el Congreso Forma y Simetría, Arte y ciencia. Buenos Aires. Recuperado de: http://www.mi.sanu.ac.rs/ vismath/BA2007/sym42.pdf Inberty, M. (2012). 
¿Podemos hablar seriamente de narratividad en música? Blelín SACCoM, 4 (2), p. 8-20. Recuperado de http://www.saccom.org.ar/saccom/boletin/v4n2/

imberty_podemos_hablar_seriamente_de_narratividad_en_musica_1997.pdf Joost, G. y Scheuermann, A. (2006). Audiovisual Rhetoric: A Metatheoretical Approach to Design. Design Research Society. International Conference in Lisbon. IADE. Recuperado de:

http://www.iade.pt/drs2006/wonderground/proceedings/fullpapers/DRS2006_0031.pdf Joost, G.; Buchmüller, S.; y Englert, R. (2008). Audio-visual Rhetoric: Visualizing the Pattern Language of Film. Available from Sheffield Hallam University Research Archive (SHURA). Recuperado de http://shura.shu.ac.uk/556/

\section{Recursos Audiovisuales}

Curtis, Hanson. (1994). The River Wild. Mendes, Sam. (1999). American beauty. Woo, John. (2002). Hostage.

\section{Abstract:}

Or musical form? The question is not capricious. There is a "family resemblance" between the mechanisms from which we know the world and at the same time we recognize ourselves in it. This paper assumes that design, as any act of knowledge, is not a field that is merely "inspired". All creation implies, among other things, that the designer taps into a repertoire of processing operations that are expressed -as Jacques Durand could see in the ancient figures of rhetoric. First we will try to articulate this repertoire of operations with related concepts such as proto-narrative and degree 0 . Second, a "table of elements" that accounts for all possible operations will be outlined. In addition, we'll make special emphasis on the rhetoric of audiovisual and generating resources of musical form. This study is part of a tradition that has its origins in the work of Roland Barthes about the rhetoric of the image and aims to contribute to the construction of a meta-theory of design and creation processes.

Key words:

audiovisual rethoric - musical rethoric - degree 0 - Roland Barthes - Jaques Durand - image - sound - creativity design.

\section{Resumo:}

O que têm em comum coisas tão diferentes como as mutações cromossó- micas, as operações de superposição da simetria ou a forma musical? Existe uma semelhança entre os mecanismos a partir dos quais conhecemos o mundo e ao mesmo tempo nos reconhecemos nele. Este trabalho supõe que o design, como todo ato de conhecimento, não é um campo meramente inspirado. Toda criação implica entrar num repertório de operações de transformação que se expressam, tal como Jaques Durand analisou, nas antigas figuras da retórica. Se intenta, articular este repertorio de operações com conceitos conexos como envolvente, proto-narrativa e grau 0. Em segundo lugar, se desenhará uma tabela de elementos que mostre a totalidade das operações possíveis. Também se fará especial ênfase na retórica do audiovisual e os recursos geradores da forma musical. Este estudo se inscreve numa tradição que tem origem nos trabalhos de Roland Barthes sobre a retórica da imagem e aspira a contribuir à construção de uma meta - teoria do design e os processos de criação.

\section{Palavras chave:}


retórica audiovisual - retórica musical - grau zero - Roland Barthes - Jaques Durand - imagem - sonido criatividade - design.

Creación y operaciones de transformación: aportes para una retórica del diseño fue publicado de la página 65 a página83 en Cuadernos del Centro de Estudios de Diseño y Comunicación Nº 64 